Taxonomía y sistemática

\title{
Primer registro de Marasmiellus volvatus (Marasmiaceae: Agaricomycetes) en Argentina, una especie poco conocida
}

\author{
First record of Marasmiellus volvatus (Marasmiaceae: Agaricomycetes) in Argentina, a rarely \\ known species
}

\author{
Nicolás Niveiro $^{\mathrm{a}, *}$, Natalia A. Ramírez ${ }^{\mathrm{a}}$, Orlando F. Popoff ${ }^{\mathrm{a}}$ y Edgardo O. Albertó ${ }^{\mathrm{b}}$ \\ ${ }^{a}$ Instituto de Botánica del Nordeste, Universidad Nacional del Nordeste, Consejo Nacional de Investigaciones Científicas y Técnicas. Sargento Cabral 2131, \\ CC 209, Corrientes Capital, 3400, Argentina \\ ${ }^{\mathrm{b}}$ Instituto de Investigaciones Biotecnológicas, Instituto Tecnológico de Chascomús. Universidad Nacional de San Martín, Consejo Nacional de Investigaciones \\ Científicas y Técnicas. Camino circ. Laguna km. 6, Chascomús, Buenos Aires, 7130, Argentina \\ Recibido el 7 de abril de 2014; aceptado el 27 de febrero de 2015 \\ Disponible en Internet el 29 de julio de 2015
}

\section{Resumen}

Marasmiellus volvatus es una especie pequeña, pero llamativa, que se encuentra pocas veces registrada en el mundo. Se caracteriza por sus basidiomas completamente blancos, píleo sulcado, láminas sin intervenosas y estípite con una volva en la base. La especie más próxima, M. coilobasis, se diferencia por la presencia de láminas con intervenosas y por los pleurocistidios y esporas de mayor tamaño. Esta es la primera cita de $M$. volvatus para Argentina, y es el registro más austral de la especie. En este trabajo se describe e ilustra a $M$. volvatus y se presenta una clave de identificación de la sección Candidi con las especies más próximas.

Derechos Reservados (C) 2015 Universidad Nacional Autónoma de México, Instituto de Biología. Este es un artículo de acceso abierto distribuido bajo los términos de la Licencia Creative Commons CC BY-NC-ND 4.0.

Palabras clave: Fungi; Basidiomycota; Agaricales; Tricholomataceae; Candidi

\begin{abstract}
Marasmiellus volvatus is a small but striking species which was found only few times in the world. It is characterized by its white basidiomata, sulcate pileus, lacking intervenous lamellae and stipe with a volva at the base. Marasmiellus coilobasis is a close species which differs from $M$. volvatus because of the presence of intervenous lamellae and larger pleurocystidia and spores. Marasmiellus volvatus is described and illustrated. Also an identification key to the species of section Candidi is presented. This is the first report of M. volvatus in Argentina and is the southernmost record reported of the species.

All Rights Reserved @ 2015 Universidad Nacional Autónoma de México, Instituto de Biología. This is an open access item distributed under the Creative Commons CC License BY-NC-ND 4.0.

Keywords: Fungi; Basidiomycota; Agaricales; Tricholomataceae; Candidi

\section{Introducción}

El género Marasmiellus Murrill se caracteriza por sus basidiomas marasmioides, colibioides o pleurotoides, pequeños, con

\footnotetext{
* Autor para correspondencia.

Correo electrónico: niconiveiro@gmail.com (N. Niveiro).

La revisión por pares es responsabilidad de la Universidad Nacional Autónoma de México.
}

contexto muy delgado, pero duros al tacto. El píleo es convexo, generalmente depreso a umbilicado, desde blanco a castaño oscuro, presenta láminas anexas a adnatas, muchas veces con intervenosas; el estípite es central, excéntrico o lateral, e inserto en el sustrato. Los caracteres microscópicos distintivos son las esporas lisas, inamiloides y una pileipellis formada por células con estructuras «rameales», por lo menos en algunos sectores, o por hifas diverticuladas (Largent y Baroni, 1988; Singer, 1973, 1986), aunque la sección Candidi puede presentar elementos no ramificados en su pileipellis (Singer, 1973).
\end{abstract}


Es un género que en el mundo cuenta con más de 250 especies (Kirk, Cannon, Minter y Stalpers, 2008), y es muy diverso en la región Neotropical (Singer, 1973). Para Argentina se encuentran registradas 45 especies, principalmente para las provincias del norte del país (Niveiro y Albertó, 2012). En Argentina es un género que fue estudiado por Singer y Digilio (1952), Singer (1952, 1953, 1954, 1969, 1989), Spegazzini (1899), Raithelhuber (2004) y Wright y Wright (2005), pero es indiscutible que el trabajo más relevante del género es la monografía de todas las especies neotropicales llevada a cabo por Singer (1973).

En el marco de recientes colecciones realizadas en el norte de Argentina se identificó a Marasmiellus volvatus Singer, una especie descrita originalmente para la Amazonia brasilera por Singer (1989) y pocas veces citada desde su descubrimiento (Franco-Molano, Vasco-Palacios, López-Quintero y Boekhout, 2005; GBIF, 2014). El objetivo del presente trabajo es describir los materiales argentinos de $M$. volvatus, ilustrarlos y comentar acerca de las especies próximas.

\section{Materiales y métodos}

El material coleccionado fue fotografiado y descrito macroscópicamente in situ, utilizando la terminología propuesta por Largent (1986), Lodge et al. (2004) y Wright y Albertó (2002). Para las observaciones microscópicas (elementos del revestimiento pileico, del contexto del píleo y del estípite, de las láminas, basidiosporas, basidios, cistidios, etc.) se realizaron cortes a mano alzada montados en $\mathrm{KOH} 5 \%$ teñidos con floxina acuosa al $1 \%$ y reactivo de Melzer (Wright y Albertó, 2002). Las medidas de todas las estructuras se dan en rangos. En el caso particular de las esporas, se utilizan las siguientes abreviaturas: $\mathrm{x}=$ media aritmética del largo $\mathrm{y}$ ancho de las esporas, $\mathrm{Q}=$ rango de variación en el coeficiente entre la longitud y el ancho de las esporas, $\mathrm{Qx}=$ media de los valores de $\mathrm{Q}, \mathrm{n}=$ número de esporas medidas. Para los colores se utilizó la terminología propuesta por Kornerup y Wanscher (1978). Los autores de los nombres científicos se indican de acuerdo con el Index Fungorum-Authors of Fungal Names (2014), y las siglas de los herbarios según Thiers (2014). El material recolectado está depositado en el herbario del Instituto de Botánica del Nordeste (CTES).

\section{Descripción}

Clave de las especies neotropicales de Marasmiellus sección Candidi:
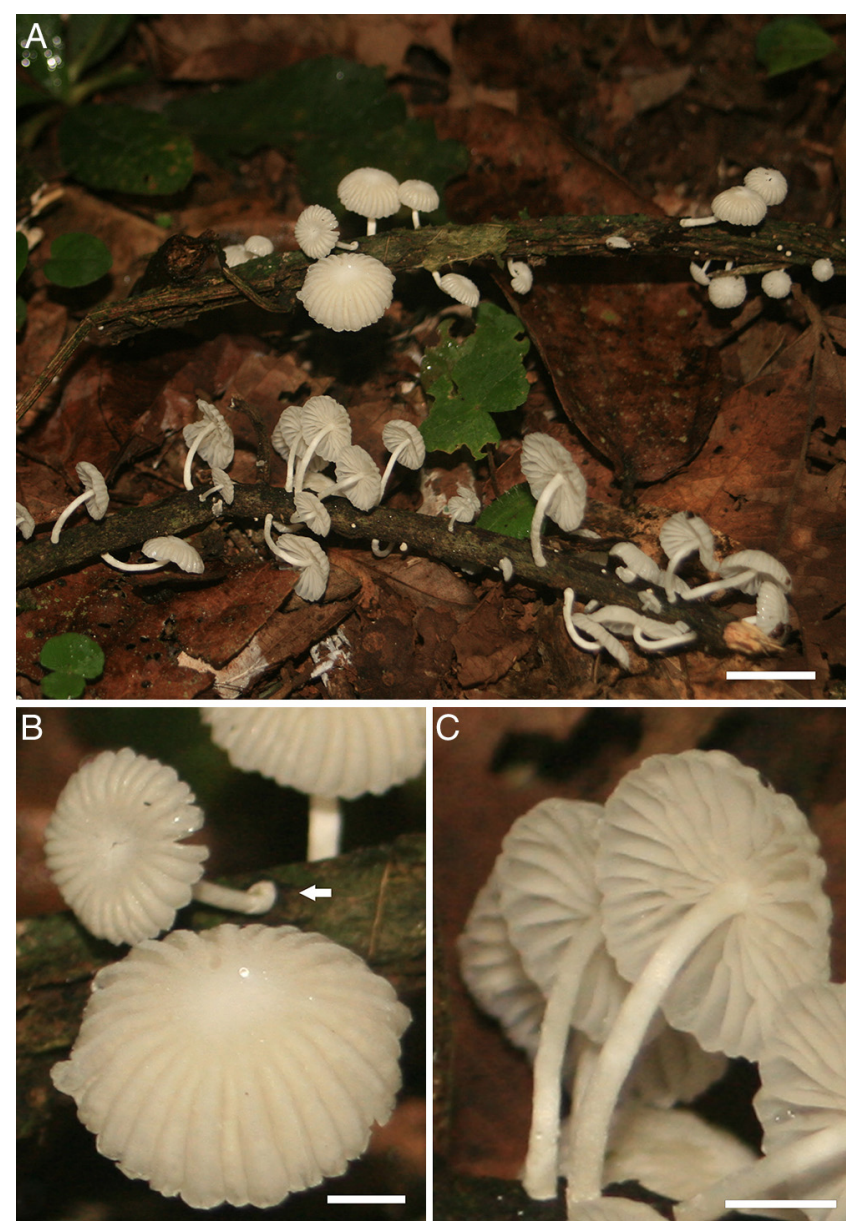

Figura 1. Caracteres macroscópicos de Marasmiellus volvatus [Niveiro y Popoff 2473 (CTES)]: A, aspecto general; B, detalle de la superficie del píleo y volva en la base del estípite (indicada con la flecha); $\mathrm{C}$, detalle de las láminas. Escala: $\mathrm{A}=20 \mathrm{~cm}$, B y $\mathrm{C}=5 \mathrm{~cm}$.

Marasmiellus volvatus Singer, Fieldiana Botany 21: 37.1989 (figs. 1-2)

Basidiomas colibioides, gregarios, xilófagos. Píleo 10$25 \mathrm{~mm}$ de diámetro, convexo, centro levemente depreso, blanco puro en el centro, blanco a blanco crémeo hacia los márgenes, superficie seca, glabra, margen sulcado hasta cerca del centro, levemente incurvado. Contexto delgado, blanco, membranáceo. Olor y sabor indiferenciados. Himenóforo lamelado. Láminas regulares, anexas a libres, blancas, distantes, delgadas, con margen entero, con lamélulas, sin intervenosas. Estípite central, 7-20 × 1-2 mm, cilíndrico, curvo, blanco, finamente fibriloso,

1. Base del estípite con estructura en forma de copa, semejante a una volva, o con forma de anillo ínfero membranáceo, sobre todo, en ejemplares jóvenes. ........2

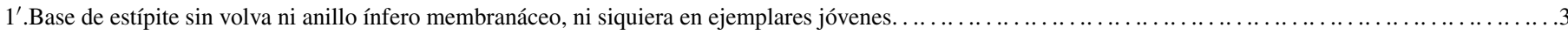

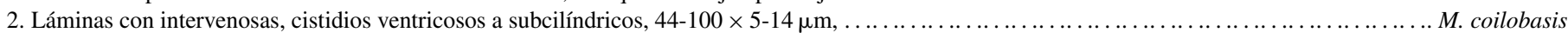

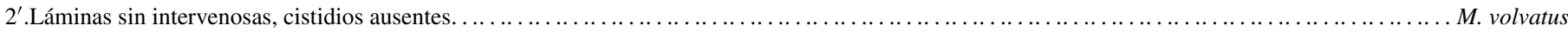

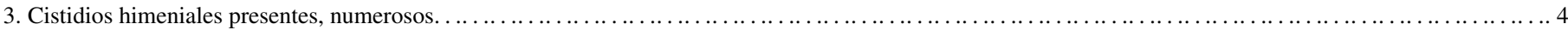

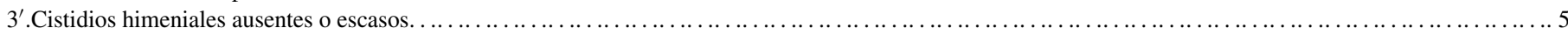

4. Basidiomas no tienden a ennegrecerse cuando secos. Píleo 6-20 mm de diámetro, estípite $10-20 \mathrm{~mm}$ de largo. ............................. albofuscus

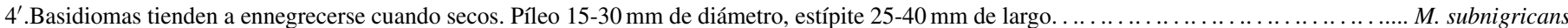

5. Estípite sólido, $0.7-14 \times 0.1-0.7 \mathrm{~mm}$, pleurocistidios ausentes, queilocistidios cilíndricos filamentosos, dispersos. ....................... helminthocystis

$5^{\prime}$.Estípite fistuloso, finalmente hueco, 4-30 $\times 0.7-1.8 \mathrm{~mm}$, pleurocistidios escasos, queilocistidios filamentosos a subcilíndricos.................. albuscorticis 
A

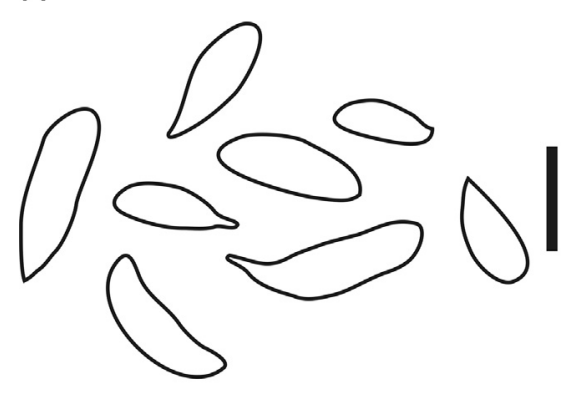

B

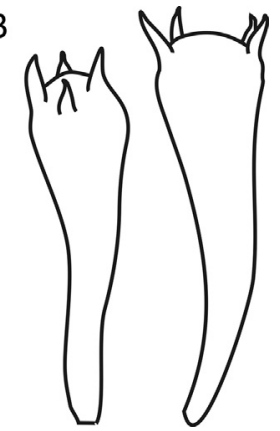

Figura 2. Caracteres microscópicos de Marasmiellus volvatus [Niveiro y Popoff 2473 (CTES)]: A, esporas; B, basidios. Escala $=10 \mu \mathrm{m}$.

fibrillas blancas, seco. Base del estípite con una volva, membranosa, angosta, adpresa, con el margen levemente levantado, blanca, bien desarrollada en ejemplares jóvenes; en ejemplares más viejos se suele perder. Anillo ausente. Esporada blanca.

Basidiosporas 11-19 $\times 3-6 \mu \mathrm{m}, \mathrm{x}=16.6 \times 5.2 \mu \mathrm{m}, \mathrm{Q}=2.5$ 4.2, $\mathrm{Qx}=3.1, \mathrm{n}=25$, fusiformes a naviculares, hialinas, lisas, inamiloides. Basidios 30-37 × 9-10 $\mu \mathrm{m}$, tetraspóricos, claviformes, de paredes delgadas, hialinos. Pleurocistidios ausentes. Queilocistidios ausentes. Trama himenoforal subregular, formada por hifas de 5-6 $\mu \mathrm{m}$ de diámetro. Pileipellis en un cutis, formada por hifas delgadas de 3-4 $\mu \mathrm{m}$ de diámetro, no ramificadas ni diverticuladas; no se observaron elementos rameales ni hifas diverticuladas aisladas. Fíbulas presentes.

\section{Resumen taxonómico}

Argentina. Provincia Misiones, Departamento General Belgrano, Reserva Natural Estricta San Antonio, en selva con ejemplares dispersos de Araucaria angustifolia, $26^{\circ} 01^{\prime} 35^{\prime \prime} \mathrm{S}$, 53 47'39.5” O, 523 m snm. 28.V.2009, leg. Niveiro y Michlig 1434 (CTES). Departamento Guaraní, Reserva de Biosfera Yabotí, Jardín Botánico Yasí Yateré, $27^{\circ} 14^{\prime} 38.5^{\prime}$ S, $54^{\circ} 01^{\prime} 52^{\prime \prime}$ O, $184 \mathrm{~m}$ snm. 18.IV.2011, leg. Niveiro y Popoff 2473 (CTES).

\section{Comentarios taxonómicos}

Especie de distribución neotropical, conocida para Brasil (Singer, 1988, 1989); Venezuela (Singer, 1988); Colombia (Franco-Molano et al., 2005); Costa Rica y Belice (GBIF, 2014). Los materiales aquí descritos representan el primer registro para Argentina, y es el registro más austral de la especie. Esta especie se caracteriza por sus basidiomas completamente blancos, píleo sulcado y estípite con una volva en la base (Singer, 1988, 1989). Pertenece a la sección Candidi por su pileipellis formada por un cutis de elementos no diverticulados ni estructuras tipo «rameales» y esporas grandes que superan los $10 \mu \mathrm{m}$ (Singer, 1973).

De los materiales analizados se observó que las medidas de las esporas presentaron un rango mayor a las medidas citadas por Singer $(1989)(10.5-13 \times 3.5-4 \mu \mathrm{m})$ y Franco-Molano et al. (2005) $(10-12 \times 3.5 \mu \mathrm{m})$, siendo similares en el límite inferior, pero llegando a ser más grandes. Sin embargo, Singer (1988), en una primera descripción de la especie cita esporas más grandes $(10.5-19 \times 4-6 \mu \mathrm{m})$, similares a las obtenidas en este trabajo.
Esto demuestra la amplia variedad del tamaño de las esporas que presenta esta especie, al igual que lo observado por Guzmán (2004) para Marasmiellus cubensis (Berk. y M. A. Curtis) Singer.

Una especie muy similar y de distribución también neotropical es M. coilobasis (Berk.) Singer. Esta presenta el píleo de color blanco, con margen sulcado y la base del estípite con una estructura similar a una volva, al igual que $M$. volvatus. Sin embargo, $M$. coilobasis se diferencia en que presenta láminas con intervenosas, presenta cistidios ventricosos a subcilíndricos y tiene esporas de mayor tamaño: esporas de 11.5-19 × 4-7.8 (8.2) $\mu \mathrm{m}$ provenientes de basidios tetraspóricos, y esporas de (17-) 1925 (-27.5) × 5.5-6.2 $\mu \mathrm{m}$ de basidios bispóricos (Singer, 1973). Marasmiellus albofuscus (Berk \& Curtis) Singer y M. subnigricans (Murrill) Singer se diferencian por no presentar en la base del estípite una estructura del tipo volva, y por poseer láminas con intervenosas y numerosos pleurocistidios (Singer, 1973).

Otra especie próxima descrita para la Amazonia es Marasmiellus helminthocystis Singer, macroscópicamente muy similar a $M$. volvatus, pero se diferencia en que esta especie no presenta una volva y sus pileocistidios son filamentosos (Singer, 1989). Marasmiellus albuscorticis (Secr.) Singer también es otra especie similar que se encuentra extensamente distribuida en zonas templadas del hemisferio Norte. Se diferencia en que presenta el centro del píleo con tintes amarillentos o crémeo rosados, el margen puede ser sulcado o no, y no presenta una estructura en forma de copa en la base del estípite, aunque puede formar un disco basal flocoso de hifas fuertemente adheridas al sustrato (Singer, 1973).

Marasmiellus cubensis, especie conocida para Venezuela y el Caribe, presenta caracteres similares, como la presencia de una volva delicada en la base del estípite y esporas de gran tamaño (16-) 18-19 (-20) × 5-6 (-7) ㅆm (Guzmán, 2004). Sin embargo, esta especie se diferencia por su pileipellis con estructuras rameales bien definidas, y esporas más grandes, lo que la ubica dentro de la sección Tricolores (Pegler, 1983; Singer, 1973).

\section{Agradecimientos}

Los autores agradecen al Ministerio de Ecología, Recursos Naturales Renovables y Turismo de la provincia de Misiones por los permisos de colección. Este trabajo fue financiado por el MERNRyT Proyecto Regional Araucaria XXI, Bosque Atlántico-AECID, por la Myndel Botanical Foundation, la Secretaría General de Ciencia y Técnica, Universidad Nacional del Nordeste y por el Consejo Nacional de Investigaciones Científicas y Técnicas.

\section{Referencias}

Franco-Molano, A. E., Vasco-Palacios, A. M., López-Quintero, C. A. y Boekhout, T. (2005). Macrohongos de la región del Medio Caquetá, Colombia. Guía de campo. Medellín: Multiprensos, Ltda.

GBIF (Global Biodiversity Information Facility). (2014) [consultado 25 Mar2014]. Disponible en: http://www.gbif.org/occurrence/ search?taxon key $=3321124$ 
Guzmán, G. (2004). Los hongos de la península de Yucatán (México). V. Nuevas observaciones y nuevos registros. Revista Mexicana de Micología, 18, 7-12.

Index Fungorum-Authors of Fungal Names. (2014) [consultado 25 Mar 2014]. Disponible en: http://www.indexfungorum.org/names/ AuthorsOfFungalNames.asp

Kirk, P. M., Cannon, P. F., Minter, D. W. y Stalpers, J. A. (Eds.). (2008). Ainsworth \& Bisby's Dictionary of the Fungi. Wallingford: CABI Publishing.

Kornerup, A. y Wanscher, J. H. (1978). Methuen Handbook of Colour (3th ed.). London: Eyre Methuen.

Largent, D. L. (1986). How to identify mushrooms to genus I: Macroscopic features. Eureka: Mad River Press.

Largent, D. L. y Baroni, T. J. (1988). How to identify mushrooms to genus VI: Modern genera. Eureka: Mad River Press.

Lodge, J., Ammirati, J. F., O’Dell, T. E., Mueller, G. M., Huhndorf, S. M., Wang, C. J., et al. (2004). Terrestrial and lignicolous macrofungi. En G. M. Mueller et al. (Eds.), Biodiversity of Fungi. Inventory and Monitoring Methods (10th ed., pp. 127-172). San Diego: Elsevier Academic Press.

Niveiro, N. y Albertó, E. O. (2012). Checklist of the Argentine Agaricales 4. Tricholomatacea and Polyporaceae. Mycotaxon, 121, 499-500. doi: http://dx.doi.org/10.5248/121.499

Pegler, D. N. (1983). Agaric flora of Lesser Antilles. Kew Bulletin Additional Series, 9, 1-668.

Raithelhuber, J. (2004). Nueva Flora Micológica Argentina. Stuttgart: Mycosur.

Singer, R. (1952). The agarics of the Argentine sector of the Tierra del Fuego and limitrophous regions of Magallanes area. Part 1. White and pink spored groups. Sydowia, 6, 165-226.
Singer, R. (1953). Quelques Agarics nouveaux de l'Argentine. Revue de Myсоlogie, Paris, 18, 3-23.

Singer, R. (1954). Agaricales von Nahuel Huapi. Sydowia, 8, 100-157.

Singer, R. (1969). Mycoflora Australis. Beihefte zur Nova Hedwigia, 29 $1-405$.

Singer, R. (1973). The genera Marasmiellus, Crepidotus and Simocybe in the Neotropics. Beihefte zur Nova Hedwigia, 44, 1-517.

Singer, R. (1986). The Agaricales in Modern Taxonomy (4th ed.). Koenigstein: Koeltz Scientific Books.

Singer, R. (1988). Agaricales mit volva. Zeitschrift fur Mykologie, 54, 41-44.

Singer, R. (1989). New taxa and new combinations of Agaricales (Diagnoses fungorum novorum Agaricalium IV). Fieldiana Botany, 21, 1-133.

Singer, R. y Digilio, A. P. L. (1952). Pródromo de la flora agaricina argentina. Lilloa, 25, 6-461.

Spegazzini, C. (1899). Fungi argentini novi v. critici. Anales del Museo Nacional de Buenos Aires, 6, 6-365 [1898].

Thiers, B. (2014, continuously updated) Index Herbariorum: a global directory of public herbaria and associated staff. New York Botanical Garden's Virtual Herbarium [consultado 25 Mar 2014]. Disponible en: http://sweetgum.nybg.org/ih/

Wright, J. E. y Albertó, E. O. (2002). Guía de los hongos de la Región Pampeana I. Hongos con laminillas. Buenos Aires: L.O.L.A

Wright, J. E. y Wright, E. (2005). Checklist of the mycobiota of Iguazu National Park (Misiones, Argentina). Boletín de la Sociedad Argentina de Botánica, $40,23-44$. 